\title{
A state of art on 3D face modeling approaches
}

\begin{abstract}
The model-based face recognition approach is based on constructing a model of the human face, which is able to capture the facial variations. The basic knowledge of human face is highly utilized to create the model. In this paper, we try to address and review the approaches and techniques used in the last ten years for modeling the human face in the 3D domain. Our discussion also shows the pros and cons of each approach used in the 3D face modeling.
\end{abstract}

Keyword: 3D dense , 3D face modeling, 3D morphable , Face recognition , face shape 